\title{
Subintimal Angioplasty in the Treatment of Chronic Lower Limb Ischemia
}

\author{
Sung Ki Cho, MD ${ }^{1}$ \\ Young Soo Do, MD ${ }^{1}$ \\ Sung Wook Shin, MD' \\ Kwang Bo Park, MD ${ }^{1}$ \\ Dong-lk Kim, MD² \\ Young Wook Kim, MD² \\ Duk-Kyung Kim, MD \\ Sung Wook Choo, MD' \\ In Wook Choo, MD ${ }^{1}$
}

\section{Index terms :}

Arteries, extremities

Arteries, stenosis or obstruction

Arteries, subintimal angioplasty

Korean J Radiol 2006; 7:131-138 Received October 4, 2005; accepted after revision December 9, 2005.

Departments of ${ }^{1}$ Radiology, ${ }^{2}$ Surgery, and ${ }^{3}$ Internal Medicine, Samsung Medical Center, Sungkyunkwan University School of Medicine, Seoul 135-710, Korea

Address reprint requests to: Young Soo Do, MD, Department of Radiology and Center for Imaging Science, Samsung Medical Center, Sungkyunkwan University School of Medicine, 50 llwon-dong, Kangnam-gu, Seoul 135-710, Korea.

Tel. (822) $3410-0515$

Fax. (822) 3410-2559

e-mail: ysdo@smc.samsung.co.kr
Objective: To present our experience with subintimal angioplasty (SA) for treatment of chronic lower limb ischemia (CLLI) and to assess its effectiveness and durability.

Materials and Methods: From April 2003 through June 2005, we treated 40 limbs in 36 patients with CLLI by SA. Balloons with or without secondary stent placement appropriate in size to the occluded arteries were used for SA of all lesions, except for iliac lesions where primary stent placement was done. The patients were followed for 1-23 months by clinical examination and color Doppler ultrasound and/or CT angiography. Technical results and outcomes were retrospectively evaluated. The presence of a steep learning curve for performance of SA was also evaluated. Primary and secondary patencies were determined using Kaplan-Meier analysis.

Results: Technical success was achieved in $32(80 \%)$ of 40 limbs. There was no statistical difference between technical success rates of $75 \%(18 / 24)$ during the first year and $88 \%(14 / 16)$ thereafter. There were four complications $(10 \%)$ in 40 procedures; two arterial perforations, one pseudoaneurysm at the puncture site, and one delayed hematoma at the SA site. Excluding initial technical failures, the primary patency rates at six and 12 months were $68 \%$ and $55 \%$, respectively. Secondary patency rates at six and 12 months were $73 \%$ and $59 \%$, respectively.

Conclusion: Subintimal angioplasty can be accomplished with a high technical success rate. It should be attempted in patients with CLLI as an alternative to more extended surgery, or when surgical treatment is not recommended due to comorbidity or an unfavorable disease pattern.

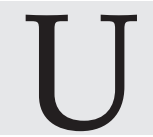

nintentional subintimal or extraluminal dissection of the arterial wall occurs frequently and is usually without sequelae, even if the vessel is perforated (1). Until recently, vessel dissection was regarded as an indication to abandon angioplasty and was a principal cause of recanalization failure. However, Bolia et al. (2) observed that a successful outcome could be achieved if the false lumen (created by the accidental subintimal passage) was pursued, with re-entry into the true lumen beyond the distal extent of the occlusion.

Since the first application of subintimal angioplasty (SA) for the treatment of occluded femoropopliteal arteries (2), promising results have been reported and the application of SA has been expanded to also include the iliac and crural arteries (321). Nevertheless, the technique has not yet gained wide acceptance because of technical difficulties and disappointing patency rates in many series $(6,8,11,14)$. There is a steep learning curve, as well as considerable skepticism regarding the procedure. 


\section{Cho et al.}

Almost all of the published studies have come from European centers, and it is only recently that studies from the United States have been reported. Therefore, it seems that more information from different institutions is required to properly identify the potential benefits and problems of this technique.

The purpose of this study was to present our experience with SA for chronic lower limb ischemia. Critical limb ischemia (pain at rest, ulcer, and gangrene) and lifestylelimiting claudication constitute the primary symptoms of chronic lower limb ischemia (11). To our knowledge, this is the first report concerning SA from outside of Europe and North America.

\section{MATERIALS AND METHODS}

From April 2003 to June 2005, 40 limbs of 36 patients with chronic lower limb ischemia were treated with SA. Three patients underwent bilateral subintimal angioplasties at separate times, one of whom underwent a repeated SA on the right lower limb due to re-occlusion of the vessel treated eight months previously. There were 32 men and four women, ranging in age from 57 to 83 years with a mean age of 70.5 years. A majority of patients had significant medical comorbidities, including hypertension $(\mathrm{n}=$ $30)$, diabetes mellitus $(n=23)$, symptomatic ischemic heart disease $(n=24)$, smoking $(n=20)$, cerebrovascular disease $(\mathrm{n}=10)$, and chronic renal failure $(\mathrm{n}=6)$.

Patient selection occurred at multidisciplinary meetings involving surgeons and interventional radiologists. Prior to committing to a specific therapeutic decision at the multidisciplinary meetings, the aortoiliac and lower extremity arteries of each patient were objectively evaluated by magnetic resonance angiography $(n=1)$, diagnostic angiography $(n=4)$, or computed tomography (CT) angiography with generation of three dimensional reconstructions $(n=35)$. SA was indicated only for patients with peripheral arterial occlusive disease. Patients with stenotic lesion were excluded. SA was undertaken as an alternative to surgery or when a patient with chronic lower limb ischemia was thought to be unsuitable for bypass surgery either because of co-morbidity or an unfavorable disease pattern.

The indications for treatment were intermittent claudication (walking distance $<300 \mathrm{~m})(\mathrm{n}=18)$, pain at rest $(\mathrm{n}=$ $2)$, ulceration ( $\mathrm{n}=11)$, gangrene $(\mathrm{n}=5)$, or a non-healing wound $(n=4)$ at the site of prior amputation. Following SA, subsequent minor amputations, including toe or transmetatarsal procedures, were planned in eight patients presenting with gangrene or a non-healing wound at the site of amputation. Below-knee amputation was planned for one patient who presented with a non-healing wound at the site of prior transtarsal amputation. The location of the arterial occlusions treated by SA included the iliac $(\mathrm{n}=$ 2), femoral $(n=10)$, femoropopliteal $(n=4)$, popliteal $(n=$ $1)$, popliteotibial $(n=2)$, tibial $(n=13)$, and femorotibial arteries $(n=1)$. The remaining seven patients had two separate occlusions at femoral and tibial locations $(\mathrm{n}=5)$, femoral and popliteotibial ones $(\mathrm{n}=1)$, and popliteal and tibial arteries $(\mathrm{n}=1)$. The length of the occlusions ranged from 2 to $34 \mathrm{~cm}$, with a mean length of $12.8 \mathrm{~cm}(<5 \mathrm{~cm}$, 12 occlusions; $5-10 \mathrm{~cm}, 9$ occlusions; $>10 \mathrm{~cm}, 19$ occlusions). The length of the occlusions in the seven patients with two separate occlusions in the target extremity were counted as the sum of the two lesions.

\section{Subintimal Angioplasty}

Before all procedures, the potential risks and benefits were explained in detail, and an informed written consent was obtained from each patient. All punctures were made using a micropuncture set (Cook, Bloomington, IN) with/without ultrasound guidance. For the two iliac subintimal angioplasties, bilateral retrograde punctures of the common femoral artery (CFA) were used. For the 38 infrainguinal subintimal angioplasties, access to 37 was made by an ipsilateral antegrade puncture of the CFA. Three of these cases needed an additional contralateral retrograde puncture of the CFA to treat a concomitant severe stenosis at the inflow of the iliac artery. One infrainguinal lesion was accessed by a contralateral retrograde puncture of the CFA to treat a concomitant severe stenosis at the inflow of the common iliac artery. Following 6 or 7 Fr arterial sheath placement, a diagnostic angiogram of the target lower extremity was obtained and recanalization procedures were attempted. In two cases of iliac occlusion, SA was used because the initial transluminal recanalization was failed.

Following administration of intravenous heparin (3,000$5,000 \mathrm{IU}, 70 \mathrm{IU} / \mathrm{kg}$ ), and an intraarterial vasodilating agent such as nitroglycerin $(100-200 \mathrm{lg})$ at the discretion of treating interventional radiologist, SA was performed using a technique similar to that described by Reekers and Bolia (5). An angled 0.035-inch hydrophilic guide wire (Terumo, Tokyo, Japan) and a supporting $5 \mathrm{Fr}$ catheter (Angled tapered catheter, Terumo, Tokyo, Japan; Davis or Van Andel catheters, Cook, Bloomington, IN) were used to create a subintimal dissection plane above the level of the occlusion. The wire then was advanced, and a loop was naturally formed at the tip of the guide wire. The loop and catheter were advanced through the subintimal plane until the occlusion was passed. A loss of resistance was often encountered as the wire reentered the true lumen of the 


\section{Subintimal Angioplasty in Chronic Lower Limb Ischemia}

native artery distal to the occlusion. Recanalization was confirmed by advancing the catheter over the guide wire beyond the point of reentry and performing an angiogram. In a case of proximal superficial femoral artery (SFA) occlusion accessed by a contralateral retrograde puncture of CFA, a subintimal dissection plane was successfully made by a simple combination of a guide wire and a $5 \mathrm{Fr}$ angled tapered catheter, without requirement of balloon stabilization recommended in a previous study (5). The recanalized segment was then balloon dilated at 8 to 10 atmospheres for 5 to 10 seconds with an appropriately sized angioplasty balloon (5- or 6-mm balloon for femoral lesions, 4- or 5-mm balloon for popliteal lesions, and 3-mm balloon for tibial lesions), depending on the diameter of the adjacent normal artery. Primary stent placement (SMART control, Cordis, Miami, FL) and subsequent postdilatation using a balloon catheter 1-mm less in diameter were used in two iliac subintimal angioplasties. In both of these, predilatation of the subintimal tract with a balloon catheter was not needed because the stent delivery system easily passed along the tract. Other significant stenotic arterial lesions (two in a contralateral iliac artery, four in an ipsilateral iliac artery, nine in a SFA, two in a popliteal artery, and four in a tibial artery) that were identified outside the area of subintimal angioplasty were treated concurrently at the time of the procedure, either with adjunctive balloon angioplasty or stent placement. Patency, adequacy of flow, and preservation of runoff were confirmed on completion angiography. If flow was impaired, repeat balloon dilatations were performed throughout the segment, using a higher inflation pressures of up to 12 atmospheres and for a longer time, $30-60$ seconds. Two cases of femoral occlusions required self-expandable stent placement (SMART control, Cordis, Miami, FL) at the entry site of SA because flow-limiting stenosis was still observed following repeated balloon angioplasties. Unless contraindicated, all patients received aspirin (100 mg daily) and clopidogrel (75 mg daily) at the time of the procedure and thereafter. All patients were closely followed for immediate and delayed complications. Because ankle brachial index (ABI) measurement was performed at the discretion of the examining vascular surgeon, pre- and post-procedural ABIs were not available in all patients. The duration of follow-up was one month to 23 months, with a mean of 10 months (median, eight months). Follow-up of the patients included clinical examinations and imaging studies of color Doppler US and/or CT angiography at three to six month intervals.

\section{Definition and Analysis}

Technical success was defined as recanalization of the occluded arterial segment, with good antegrade flow on the completion angiography. Clinical success was defined as resolution or improvement of claudication, relief of pain at rest, healing of ulcers, or healing of minor amputations required for gangrene and non-healing wounds at the site of previous amputation. Patency was defined as the absence of occlusion and less than $50 \%$ stenosis in the treated arterial segment.

Institutional review board approval for the required retrospective review of patient medical and imaging records was obtained. We retrospectively evaluated technical results and outcomes for SA. The primary and secondary patency rates were calculated using KaplanMeier analysis. In addition, we compared the results during the first year and thereafter to evaluate whether there was a steep learning curve for SA, and this comparison was statistically analyzed using Fisher's exact test.

\section{RESULTS}

Technical success for SA was achieved in $32(80 \%)$ of 40 limbs in 28 (78\%) of 36 patients (Fig. 1). Of eight technical failures, seven resulted from an inability to reenter the true lumen distal to the occlusion, and one resulted from failure to pass the wire into the subintimal plane. Two of these eight patients who failed SA were treated conservatively, due to refusal of below-knee amputation in one and an unfavorable disease pattern for surgery in the other. One patient died of acute respiratory distress syndrome unrelated to the procedure one month later. One patient required CFA-peroneal bypass surgery. Two patients underwent a below-knee amputation, and two patients required minor amputation (great toe amputation in one and transmetatarsal amputation in the other) for healing of gangrene or of the wound at the amputation site.

Clinical success was achieved in 31 (78\%) of 40 limbs in $27(75 \%)$ of 36 patients. Despite technical success, one patient underwent a below-knee amputation because his wound did not heal following SA and subsequent transmetatarsal amputation. One patient who presented with a non-healing wound at the site of prior transtarsal amputation had a successful SA and subsequent belowknee amputation. There were four complications $(10 \%)$ in 40 procedures: two arterial perforations, one pseudoaneurysm at the puncture site, and one hematoma at the SA site. One arterial perforation occurred at a collateral branch vessel during the guide wire manipulation for subintimal cannulation of the occluded peroneal artery. This was successfully treated with coil embolization. The other arterial perforation occurred at a subintimally dissected distal SFA in a patient with a long segmental SFA 


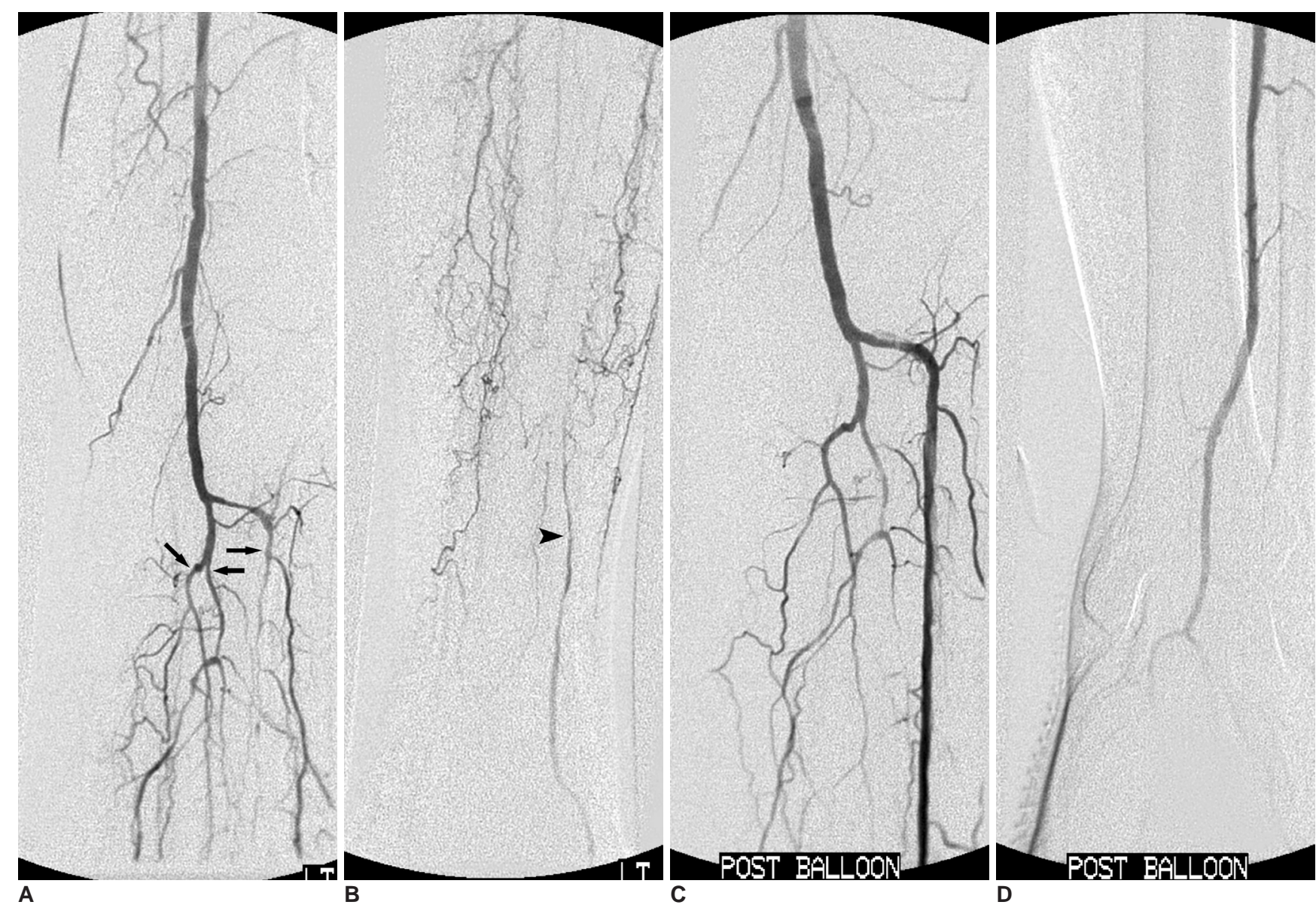

Fig. 1. A 63-year-old man who underwent subintimal angioplasty for an occluded left anterior tibial artery. There were unhealing ulcers of the 4-5th toes of left foot.

A, B. Angiograms of left lower extremity show proximal occlusions of all tibial arteries (arrows) with reconstitution of the distal anterior tibial artery (arrowhead) above the ankle.

C, D. After subintimal angioplasty of the occluded anterior tibial artery (not shown), the anterior tibial artery is successfully recanalized with good antegrade flow to the pedal artery (In figure D, the plane of view for the angiogram was right anterior oblique $16^{\circ}$ ). After SA, the ulcers healed completely and the patency of the treated left anterior tibial artery was maintained for three months until the time of investigation (not shown).

occlusion. This was treated by ultrasound guided compression, because we failed to reenter the distal true lumen. A small pseudoaneurysm with surrounding hematoma at the puncture site, which was identified on US examination the day after the procedure, spontaneously occluded during follow-up. A hematoma occurred two days after the procedure at the SA site of the posterior tibial artery. This had not been identified on US examination the day after the procedure. It resolved with conservative management using an elastic bandage.

During follow-up, arterial re-occlusion or stenosis $>50 \%$ occurred in 13 limbs of 12 patients, with the time of onset ranging from two to 19 months (mean, 7.2 months; median, six months). One patient in whom re-occlusion of the treated arteries occurred six months after SA underwent a repeat SA with clinical success. However, the arteries occluded again eight month later and the patient finally underwent femoral-popliteal bypass. Of the remaining 11 patients with arterial re-occlusion or stenosis > $50 \%$, five were asymptomatic $(n=4)$ or had improved symptoms $(\mathrm{n}=1)$, and they were followed up without further treatment (Fig. 2). Six patients with tolerable symptoms $(n=3)$ or an unfavorable disease pattern for bypass surgery $(\mathrm{n}=3)$ were treated conservatively. Two patients were lost to follow-up at three and six months, and two patients were referred to secondary hospitals at one month and three months after SA. One patient died of pneumonia at 14 months. Using a basis of intention to treat, the primary patency rates at six months and 12 months were $55 \%$ and $44 \%$, respectively, and the secondary patency rates at six months and 12 months were $58 \%$ and $47 \%$, respectively (Fig. 3). Excluding initial technical failures, the primary patency rates at six months and 12 months were $68 \%$ and $55 \%$, respectively, and the 


\section{Subintimal Angioplasty in Chronic Lower Limb Ischemia}

secondary patency rates at six months and 12 months were $\quad(p=.439)$.

$73 \%$ and $59 \%$, respectively.

When we compared the results of the 24 procedures

performed during the first year with those of the 16

procedures that followed, the technical success rates were

$75 \%$ (18 of 24 procedures) and 88\% (14 of 16

procedures), respectively, and the complication rates were

$8.3 \%$ ( 2 of 24 procedures) and $12.5 \%$ ( 2 of 16

procedures), respectively. There was no statistically signifi-

cant difference in technical success between the two groups

\section{DISCUSSION}

Theoretically, SA should have distinct advantages over intraluminal recanalization. First, the new lumen between the tunica intima and media is presumably free from atheroma and more likely to remain patent. Second, since the wire moves freely in the newly created plane, the procedure enables treatment of long segments of disease
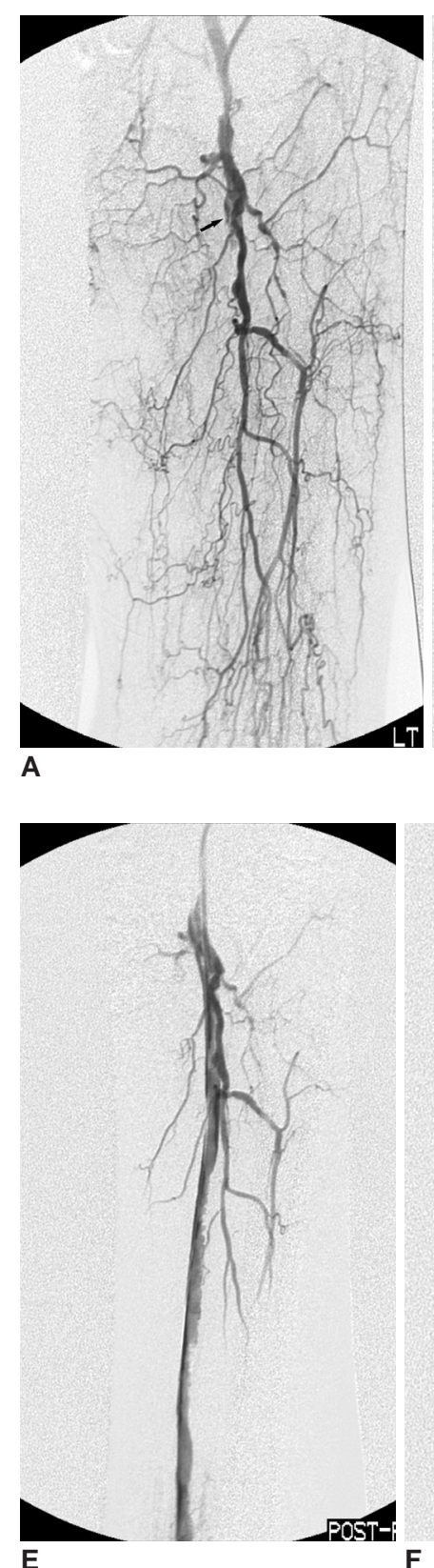
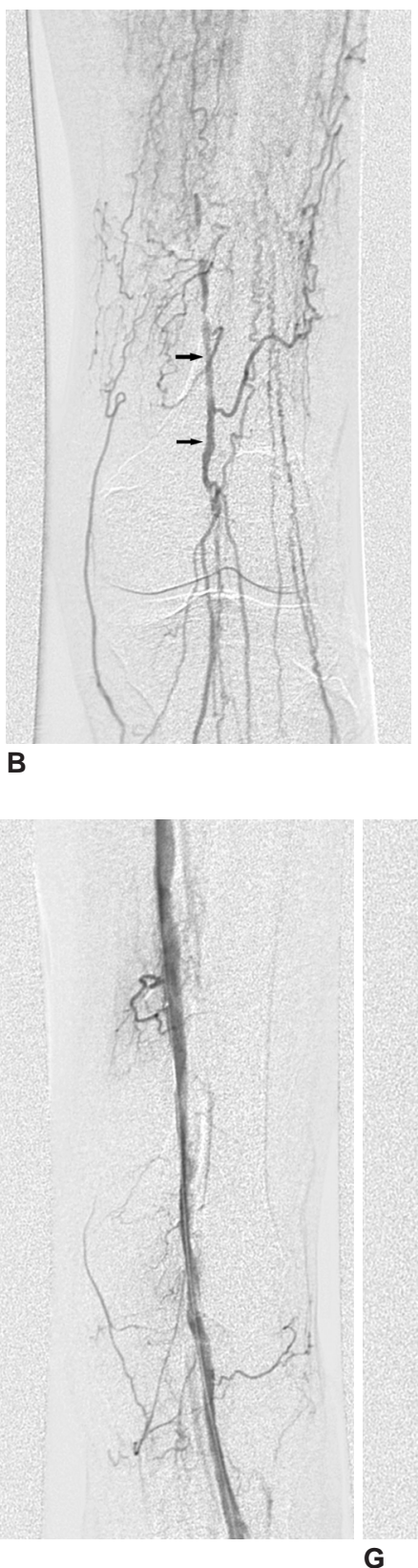

G
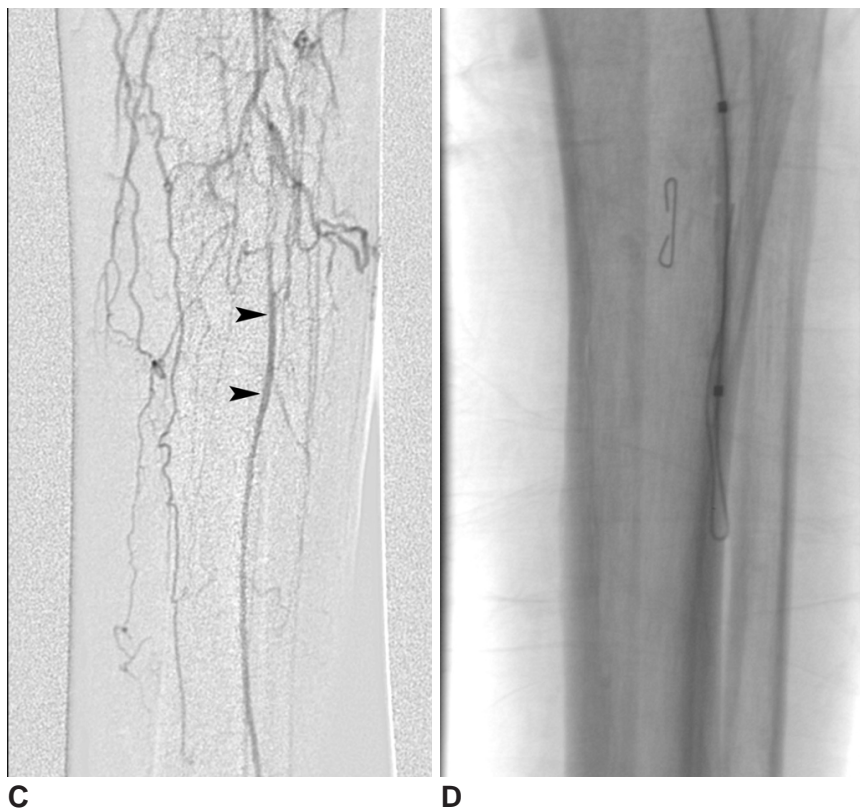

Fig. 2. A 76-year-old woman who underwent subintimal angioplasty for two separate occlusions involving the left superficial femoral and popliteotibial arteries. The lesions led to gangrene of the second toe of the left foot.

A. Preprocedural angiogram of the left lower extremity shows long-segmental occlusion of the left superficial femoral artery, extending from the origin (arrow)

B, C. After short segmental reconstitution of the left popliteal artery (arrows), downstream popliteotibial arteries are occluded with reconstitution of proximal anterior tibial artery (arrowheads).

D. The catheter and guide wire systems with a wire tip looping are passed along the subintimal plane to the occluded proximal anterior tibial artery.

E-G. After subintimal balloon dilation, postprocedural angiograms show successful recanalization of the

occluded superficial femoral artery and popliteotibial arteries with good antegrade flow to pedal artery. The patient underwent a planned metatarsophalangeal amputation three days after SA, with complete healing of the amputated site. Five months after SA, reocclusion of the treated arteries was found by color Doppler US (not shown). The patient was simply followed without any further treatment since she was asymptomatic. 


\section{Cho et al.}

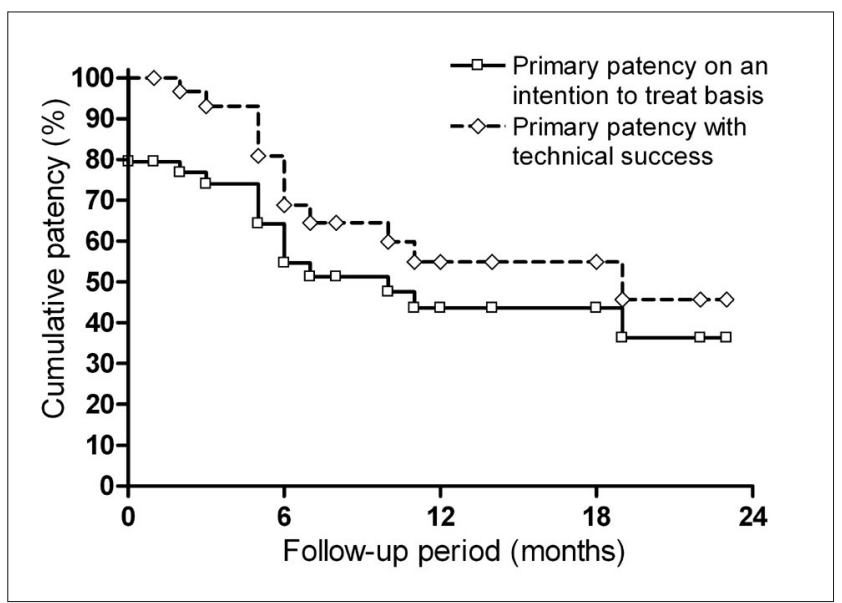

Fig. 3. A graph shows the primary patency rates of subintimal angioplasty either on the basis of intention to treat or with technical success.

with minimal additional morbidity. Third, there is a minimal risk of embolization, because plaque is not ruptured. Finally, failure to create a reentry site or to secure successful wire passage is unlikely to further jeopardize distal perfusion, because the procedure is limited to an already chronically occluded segment.

The assessment of technical success has been based on the findings of completion angiography in most studies, but, in some $(7,13,21)$, a duplex scan, ABI, clinical improvement, or their combinations have been used to determine technical success. The assessment of technical success in the present study is based on completion angiography, with a technical success rate of $80 \%$ (32 of 40 procedures), which is comparable to that $(74 \%-90 \%)$ of previous studies $(2,4,6,8-14,16,18-21)$. Reported technical failures have been attributable to an inability to enter the subintimal space, failure to advance the wire in a calcified vessel, an inability to reenter the true lumen distal to the occlusion, an inability to maintain patency of the recanalized lumen despite successful wire and catheter passage, and abandonment of the procedure so as to avoid damage to a major distal collateral $(2,6,7,11,18,20)$. Technical failures of the present study are attributed mostly to an inability to reenter the distal true lumen (7 of 8 technical failures). One technical failure resulted from an inability to create the subintimal dissection plane. Some authors have tried alternative approaches for SA, including retrograde popliteal artery puncture using ultrasound guidance (22), and simultaneous antegrade CFA and retrograde popliteal artery punctures (23). Balas et al have described open SA in cases of complete SFA occlusion where initiation of the subintimal dissection may be difficult (24). Recently, new catheter systems (Outback catheter, Perclose, Redwood City, CA) have been developed to facilitate reentry into the true lumen (25). It is hoped that variations of these methods may help overcome the technical failures of SA.

Clinical and hemodynamic patencies have been used to assess the durability of SA in the previous studies $(2,4-6$, $8-11,13,14,16,18-21)$. The assessment of clinical patency has been based on clinical symptoms alone, or on both clinical symptoms and ABI, and that of hemodynamic patency has been based on duplex US with or without ABI measurement. Based on the results of this and previous studies that present cases of arterial re-occlusion with no or unchanged symptoms $(9,13,18)$, an absence of clinical events does not necessarily indicate the anatomic patency of SA. Conversely, the presence of additional ipsilateral proximal or distal arterial lesions in some cases makes it difficult to determine the clinical patency for each repaired occlusion (6). Therefore, hemodynamic patency is believed to be better and more objective than the presence or absence of symptoms for assessing the efficacy of SA. Although it is clear that invasive arterial angiography is the best method for assessing vessel patency, almost all studies have used duplex ultrasound with or without ABI measurement for routine follow-up. Angiography has generally been reserved for confirmation of stenosis or occlusion, because it is hard to justify its use for routine follow-up. This study has assessed vessel patency based on color Doppler US and/or CT angiography. We used CT angiography, instead of diagnostic angiography, to confirm vessel patency and to check for concurrent arterial lesions which can cause recurrent symptoms. A recent study prospectively compared the accuracy of 16-detector row $\mathrm{CT}$ angiography with conventional digital subtraction angiography in the assessment of aortoiliac and lower extremity arteries in patients with peripheral arterial disease. The sensitivity and specificity of $\mathrm{CT}$ angiography with regard to detection of hemodynamically significant stenosis (> 50\% luminal narrowing) were $96 \%$ and $97 \%$, respectively (26). However, further study will be required to establish the role of CT angiography in SA. On an intention to treat basis, primary and secondary patency rates in our study are $55 \%$ and $58 \%$ at six months, and $44 \%$ and $47 \%$ at 12 months, respectively. These are somewhat disappointing, but quite comparable to those of previous studies $(4,6,8,10,11,14,27)$. If one excludes the technical failures, our results appear to be effective. Primary patency rates at six months and 12 months were $68 \%$ and $55 \%$, respectively, and secondary patency rates at six months and 12 months were $73 \%$ and $59 \%$, respectively.

In view of clinical symptoms, five of the 12 patients who 


\section{Subintimal Angioplasty in Chronic Lower Limb Ischemia}

had arterial re-occlusion or stenosis $>50 \%$ during the follow-up period were asymptomatic or had unchanged symptoms, and three patients were treated conservatively due to tolerable symptoms. Furthermore, in 2 of the 8 technical failures, gangrene or wound infection at the amputation site healed following minor amputation. Some previous studies have also reported cases of no or unchanged symptoms, despite technical failure or late arterial re-occlusion $(9,13,18)$.

The overall complication rates of SA are $5 \%$ to $20 \%$, and a majority of them can be treated conservatively or by endovascular techniques $(2,6-14,18,19)$. The complications reported are those inherent in endovascular procedures, including groin hematomas, pseudoaneurysm at the puncture site, distal embolism, arterial injury, renal insufficiency, and retroperitoneal hemorrhage. There are also those associated with the underlying health of the patient population, such as myocardial infarction. We encountered 4 complications (10\%) in 40 procedures. Endovascular treatment was required in one arterial perforation, and the other complications (one arterial perforation, one pseudoaneurysm at the puncture site, and one delayed hematoma at the SA site) were treated conservatively. We could not find the cause of the delayed hematoma that occurred at the site two days after SA. There have been two case reports of late arterial rupture associated with SA $(28,29)$, which could be a possible cause of this.

Shaw et al. (10) reported a technical success rate of $92 \%$ in their last 23 cases versus $64 \%$ in the first 25 , suggesting that SA is associated with a short learning curve. Yilmaz et al. (6) reported a similar observation. With further experience and improved patient selection for SA, our technical success rate has also improved, with technical success rates of $75 \%$ (18 of 24 ) during the first year and $88 \%$ (14 of 16) thereafter. However, this did not reach statistical significance ( $p=.439)$. Treiman (21) noted results similar to ours. Recently, Bolia has suggested that a steep learning curve is present for the first 100 to 200 procedures, and results during this time may vary (30). This suggestion might explain the above discrepancy in the steepness of the learning curve for performing SA.

Our study has several limitations. First, compared with European studies, the number of patients is relatively small. Second, retrospective data collection can create bias for determining clinical outcomes. Third, the follow-up period was not standardized. Furthermore, data were confined to the last known follow-up, so patency data could have been underestimated. Thus, we believe that a larger, prospective study with a longer follow-up period is necessary.
In conclusion, subintimal angioplasty is feasible with a high initial technical success rate, and our primary patency rate is comparable to that of other previous studies. Thus, we feel that SA should be attempted in patients with chronic lower limb ischemia, either as an alternative to surgery, or when surgical treatment is not feasible because of comorbidity or an unfavorable disease pattern.

\section{References}

1. Ashley S, Kester RC. Laser angioplasty. Br J Surg 1993;80:550551

2. Bolia A, Miles KA, Brennan J, Bell PR. Percutaneous transluminal angioplasty of occlusions of the femoral and popliteal arteries by subintimal dissection. Cardiovasc Intervent Radiol 1990;13:357-363

3. Reekers JA. Short-term results of femoropopliteal subintimal angioplasty (Br J Surg 2000;87:1361-5). Br J Surg 2001;88:887888

4. Bolia A. Percutaneous intentional extraluminal (subintimal) recanalization of crural arteries. Eur J Radiol 1998;28:199-204

5. Reekers JA, Bolia A. Percutaneous intentional extraluminal (subintimal) recanalization: how to do it yourself. Eur J Radiol 1998;28:192-198

6. Yilmaz S, Sindel T, Yegin A, Luleci E. Subintimal angioplasty of long superficial femoral artery occlusions. J Vasc Interv Radiol 2003;14:997-1010

7. Treiman GS, Whiting JH, Treiman RL, McNamara RM, Ashrafi A. Treatment of limb-threatening ischemia with percutaneous intentional extraluminal recanalization: a preliminary evaluation. J Vasc Surg 2003;38:29-35

8. Laxdal E, Jenssen GL, Pedersen G, Aune S. Subintimal angioplasty as a treatment of femoropopliteal artery occlusions. Eur J Vasc Endovasc Surg 2003;25:578-582

9. Lipsitz EC, Ohki T, Veith FJ, Suggs WD, Wain RA, Cynamon J, et al. Does subintimal angioplasty have a role in the treatment of severe lower extremity ischemia? J Vasc Surg 2003;37:386391

10. Shaw MB, DeNunzio M, Hinwood D, Nash R, Callum KG, Braithwaite BD. The results of subintimal angioplasty in a district general hospital. Eur J Vasc Endovasc Surg 2002;24:524-527

11. Tisi PV, Mirnezami A, Baker S, Tawn J, Parvin SD, Darke SG. Role of subintimal angioplasty in the treatment of chronic lower limb ischaemia. Eur J Vasc Endovasc Surg 2002;24:417-422

12. Ingle H, Nasim A, Bolia A, Fishwick G, Naylor R, Bell PR, et al. Subintimal angioplasty of isolated infragenicular vessels in lower limb ischemia: long-term results. J Endovasc Ther 2002;9:411416

13. Vraux H, Hammer F, Verhelst R, Goffette P, Vandeleene B. Subintimal angioplasty of tibial vessel occlusions in the treatment of critical limb ischaemia: mid-term results. Eur J Vasc Endovasc Surg 2000;20:441-446

14. McCarthy RJ, Neary W, Roobottom C, Tottle A, Ashley S. Short-term results of femoropopliteal subintimal angioplasty. $\mathrm{Br}$ J Surg 2000;87:1361-1365

15. Nydahl S, Hartshorne T, Bell PR, Bolia A, London NJ. Subintimal angioplasty of infrapopliteal occlusions in critically ischaemic limbs. Eur J Vasc Endovasc Surg 1997;14:212-216

16. London NJ, Srinivasan R, Naylor AR, Hartshorne T, Ratliff DA, 


\section{Cho et al.}

Bell PR, et al. Subintimal angioplasty of femoropopliteal artery occlusions: the long-term results. Eur J Vasc Surg 1994;8:148155

17. Bolia A, Bell PR. Femoropopliteal and crural artery recanalization using subintimal angioplasty. Semin Vasc Surg 1995;8:253264

18. Desgranges P, Boufi M, Lapeyre M, Tarquini G, van Laere O, Losy F, et al. Subintimal angioplasty: feasible and durable. Eur J Vasc Endovasc Surg 2004;28:138-141

19. Lazaris AM, Tsiamis AC, Fishwick G, Bolia A, Bell PR. Clinical outcome of primary infrainguinal subintimal angioplasty in diabetic patients with critical lower limb ischemia. J Endovasc Ther 2004;11:447-453

20. Florenes T, Bay D, Sandbaek G, Saetre T, Jorgensen JJ, Slagsvold CE, et al. Subintimal angioplasty in the treatment of patients with intermittent claudication: long term results. Eur J Vasc Endovasc Surg 2004;28:645-650

21. Treiman GS. Subintimal angioplasty for infrainguinal occlusive disease. Surg Clin North Am 2004;84:1365-1380, viii

22. Heenan SD, Vinnicombe SJ, Buckenham TM, Belli AM. Percutaneous transluminal angioplasty by a retrograde subintimal transpopliteal approach. Clin Radiol 1995;50:507-508

23. Spinosa DJ, Leung DA, Harthun NL, Cage DL, Fritz Angle J, Hagspiel KD, et al. Simultaneous antegrade and retrograde access for subintimal recanalization of peripheral arterial occlusion. J Vasc Interv Radiol 2003;14:1449-1454
24. Balas P, Pangratis N, Ioannou N, Milas P, Klonaris C, Masouridou E. Open subintimal angioplasty of the superficial femoral and distal arteries. J Endovasc Ther 2000;7:68-71

25. Hausegger KA, Georgieva B, Portugaller H, Tauss J, Stark G. The outback catheter: a new device for true lumen re-entry after dissection during recanalization of arterial occlusions. Cardiovasc Intervent Radiol 2004;27:26-30

26. Willmann JK, Baumert B, Schertler T, Wildermuth S, Pfammatter T, Verdun FR, et al. Aortoiliac and lower extremity arteries assessed with 16-detector row CT angiography: prospective comparison with digital subtraction angiography. Radiology 2005;236:1083-1093

27. Nadal LL, Cynamon J, Lipsitz EC, Bolia A. Subintimal angioplasty for chronic arterial occlusions. Tech Vasc Interv Radiol 2004;7:16-22

28. Wright G, Fishwick G, Naylor AR. Arterial perforation (by balloon) during subintimal angioplasty. Eur J Vasc Endovasc Surg 2004;28:108-110

29. Brookes JA, Aly S, Bishop CC. Re: arterial perforation (by balloon) during subintimal angioplasty by Dr Wright and colleagues from Leicester Royal Infirmary, Leicester, United Kingdom published in Eur J Vasc Endovasc Surg 28, 108-110 (2004). Eur J Vasc Endovasc Surg 2004;28:677-678

30. Ouriel K. A critical review of subintimal angioplasty. In:Vascular News. London: BIBA Publishing, 2003:15-16 